\title{
Research on Applications of Information Technologies in Medical Image Area
}

\author{
Juan Zhong, Honghui Lai \\ College of Information Engineering, Gannan Medical University, Ganzhou, Jiangxi 341000 \\ hunter2011@foxmail.com
}

Keywords: Information Technology; Medical Image; Data Mining; Image Enhancement; Information Sharing

\begin{abstract}
Medical image plays an important role in the modern medicine. However, the medical images also have some shortages and limitations, which can be made up by some information technologies. This paper firstly introduces the common information technologies used in medical image area, such as the data mining technology, the image enhancement technology and the information sharing technology and then elaborates the applications of the above technologies in medical image area in order to provide some references for relevant researchers.
\end{abstract}

\section{Introduction}

Modern medicine can't develop well without the support of medical images. Medical image has become an indispensable tool for clinical diagnosis, treatment and medical research. Medical image technology has integrated the theory and method of physics, mathematics, biology, medicine, materials science, information technology, computer image processing technology, and computer graphics and so on. Compared with other applications, medical image has its own characteristics and limitations. The applications of various information technologies make up the short board of medical image, which makes it play a greater role in modern medicine.

\section{Information Technologies Used in Medical Image Area}

Data Mining Technology. The data mining techniques used in medical images mainly include the artificial neural network technology, the support vector machines technology, the decisionmaking tree technology and evolutionary computation method. Artificial neural network is a new subject. Its development speed is fast, and it is mainly through the simulation of the structure of the human brain neuron to study the nonlinear prediction model. The technology can accomplish a variety of data mining tasks, including clustering, classification and association rules, etc. Artificial neural network has its own characteristics, such as strong fault tolerance and self-organization. In recent years, the technology of artificial neural network is widely used in data mining technology. The classification algorithm belongs to a new classification method, which is a comprehensive classification mining technology and association rules. The process includes two steps: first, mining related association rules is a standard association rule mining algorithm; the two is to construct a classifier based on the association rules. The support vector machine is the concrete embodiment of the structural risk minimization principle and the dimension theory. This technique transforms the input space into a high dimensional space by means of nonlinear transformation, and the optimal linear classifier is obtained from this new space. In this way, the complexity of the model and the learning ability can be found from the limited sample information to the best compromise, in order to get better generalization ability. Decision tree is a decision tree using a group of known training data, and then the model is used to predict the impact of various data. In the process of medical information, decision tree is very powerful. With the development of medical technology, decision tree algorithm has been improved and perfected. Evolutionary computation is a new method of calculation, which is based on the law of biological evolution. The method mainly includes the evolution of planning and strategy, genetic programming and algorithm. Evolutionary computation 
is a new cross subject. In recent years, the application and research of evolution has been developed, and its theoretical basis has been further improved. Evolutionary computation method can not only analyze and utilize all kinds of algorithms, but also design effective evolutionary algorithm, which can improve the existing algorithms.

Image Enhancement Technology. The features of medical image contrast, edge features and signal to noise ratio have a very important significance for the diagnosis of the disease in the analysis and reading of medical images. But all kinds of medical images will be damaged and degraded in different degrees in the process of transmission and transformation. In order to improve the contrast of the image, highlight the outline of the target, and attenuate the noise, it is necessary to enhance the image. Image enhancement is the use of various mathematical methods and transform, improve the image of the object and non-object contrast and image clarity. Here, the object is the image contained in the need to find and research content, such as lesions, tumors, shadows, etc. The purpose of image enhancement is to use a series of techniques to improve the visual effect of the image or to convert the image into a more suitable form of analysis and processing. By processing the information that has chosen to highlight the interest of some people or machines, it can inhibit some useful information and improve the use value of the image. As it is not known to the specific reasons for the image quality, the image processing method to improve the quality of the image, so it is needed to provide several ways to adapt to the specific image. Image enhancement refers to the image features such as edges, contours, contrast, etc., to facilitate observation or further analysis. The image enhancement techniques are generally divided into two categories: spatial enhancement and frequency domain. The spatial enhancement is directly in the spatial domain, and the spatial enhancement method has gray transformation and histogram transform. The effect of using a single image enhancement method is always not ideal. It needs to use a variety of image enhancement methods to enhance the medical images.

The process of the image enhancement can be described as Fig. 1. The mathematical description is as follows:

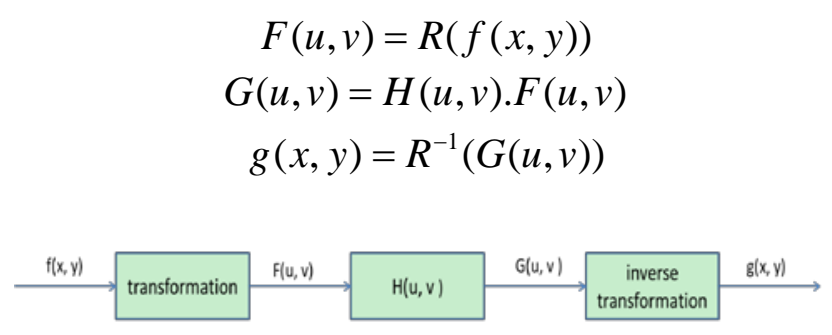

Fig. 1 Process of the Image Enhancement

Information Sharing Technology. Due to the very large amount of medical image data, the data acquisition, storage, transmission and system construction are very big different from the word processing system. The construction of medical image system needs special project. Medical image management system is often referred to as the Picture Archiving \& Communication System (PACS), which mainly includes the collection, storage, transmission and reproduction of digital medical images. The information technology, such as the PACS system and medical treatment, the nursing station system, the computer management system, the library network integration and so on, realize the resource sharing of the hospital image files. Using modern information technology, scientific classification method, organization and planning medical image data retrieval interface, easy to use, and provide intelligent, accurate and efficient fuzzy search function, the medical image data of patients, such as the name, diagnosis time and medical students and other content for retrieval. According to the type of diagnostic report, the preparation of the corresponding report template file, provide the function of the preparation of the diagnostic report. The use of personnel, with a strict operation of the query and management functions, and has a reliable database data backup and restore functions. So, we can retrieve and query a variety of forms and storage format of medical image data and information by the hospital network, realizing the medical image file management automation and modernization. 


\section{Applications of Information Technologies in Medical Image Area}

Applications of Data Mining Technology in Medical Image Area. In the study of patients with left ventricular assist device, the clinical data of patients with left ventricular assist device were retrospectively analyzed, and the existing risk score was compared. In quantitative analysis of the synergy between the right ventricular support and its main effect on the nonlinear encoding, the model of clinical reasoning can be studied more carefully. With the further development of the medical institutions, the classification and treatment of left ventricular assist device provides a valuable tool for predicting the longitudinal data. It can effectively improve the prognosis. In medical imaging reports, there is a general uncertainty, which may delay the clinical decisionmaking or cause the diagnosis error, resulting in the medical and health has a variety of adverse consequences. Compared with the filtered back projection method, the Bayesian iterative propagation gradient reconstruction algorithm is used to improve the image of the myocardial wall. This reconstruction method can quickly provide accurate attenuation map and can improve the accuracy of the corrected perfusion image in the heart. Then explain the differences between the population and the clinical, and then explain the description of the neutron group and the cluster in the optical field, and calculate the P value of the clustering, which will help us to better understand the various types of dementia. In addition, the correlation rule, algorithm and computer decision support system are mainly used to compare the specificity, sensitivity and accuracy of the classification and automatic diagnosis of renal image classification and automatic diagnosis. The results show that the combination of multiple algorithms can achieve high accuracy and good sensibility. It provides the possibility to the doctors to predict the abnormal development of the normal kidney in the decision making research.

Applications of Image Enhancement Technology in Medical Image Area. The purpose of medical image enhancement is to improve the image quality and optimize its visual effects. The method mainly includes contrast enhancement, smoothing, sharpening, pseudo color enhancement, etc. Through a large number of examples, various methods have their advantages and disadvantages, and there is no enhancement method for all images. Therefore, using the same kind of post processing technology to deal with all the images is clearly inappropriate and not ideal. We must characteristics from the image, image characteristics and various enhancement methods of choosing appropriate enhancement method, it is necessary, the several enhancement methods to combine and make them learn from each other, better display the image characteristics and good reinforcing effect is obtained. X line image is mostly black and white images, while the human eye has poor ability to distinguish gray level, the ability to distinguish color is strong, and through the pseudo color processing technology, it can improve the recognition of image features. Through a lot of experiments, we found that the image gray level distribution is reasonable and the details are outstanding, and the image can be enhanced with the image gray level distribution.

Applications of Information Sharing Technology in Medical Image Area. It is a new mode of medical and health system to use the information sharing technology to realize the medical images archive management. It has very important significance for patients and medical units. Traditional image storage requires a lot of space, the site, temperature, humidity, equipment and file management personnel have higher requirements, the image data access, but also spend more human and time. In addition, the traditional storage mode of the image data is exclusive, a piece of film is borrowed, and other people cannot borrow to read this film. To achieve image information sharing, it is able to solve the problem fundamentally. In the system, the image resources can be accessed by many people, and the electronic retrieval function can be liberated from the heavy work. Traditional CT, X-ray and other medical images are stored in the form of film, there is a large space, the film is easy to damage, loss, metamorphism, is not easy to save, search, use, increase the cost of medical disputes and medical disputes. Achieve medical image file information sharing, all images can be transmitted to the workstation, can burn into disc preservation, greatly reducing the cost of the Department, the image data is not easy to be lost, online video data at anytime and anywhere. The historical image can be retrieved, recovery, not only reduce the diagnosis and treatment of rare clinical experience and case information, but also conducive to teaching, scientific research. 
Information sharing technology is conducive to comparative analysis, to avoid duplication of inspection. In the diagnosis, due to the reasonable integration of resources, to avoid the unreasonable repeated inspection and unnecessary inspection, in the diagnostic process can be in the same time access to the data were compared and analyzed, make the diagnosis more scientific and reasonable, work efficiency also has the obviously improved. All clinical doctors and nurses have a good command of the imaging system, all kinds of images and reports can be transmitted through the hospital information network timely and quickly. Work process monitoring and management from the patient registration, inspection, report editing, report to the report of the printing and so have time record, can reflect the time, the participation of each link in the video link. Report and audit of quality management diagnosis report in addition to the superior doctors to the audit report to the customs and department director can attune to review report has been issued. It is pointed out that the information sharing technology makes the diagnosis quality continuous improvement.

\section{Conclusion}

The data mining technology is widely used in the early diagnosis of disease; the image enhancement technology makes the medical images easier to identify; the information sharing technology is the basis of digital medical image archives management. Although there are many limitations in every kind of the technology, we can synthesize various technologies to avoid the disadvantages of single information technology. Information technologies will help researchers to provide earlier and more accurate decisions in the prevention and treatment of the diseases. It will be applied more widely and even further in the medical image area to diagnose and treat the diseases of patients.

\section{References}

[1] H.W. Liu. The application of data mining technology in the medical image. Network Security Technology \& Application, 2014(4):76-78.

[2] B.H. Qiang, D. Liu, X.M. Zhu. Application progress of data mining technology in medicine imaging. Journal of Clinical Ultrasound in Medicine, 2013, 15(4):287-289.

[3] Y. Cao, W. Chen. Application of Image Enhancement in X-ray Medical Image. CT Theory and Applications, 2015, 24(4):545-551.

[4] D.H. Wang, P. Yuan, R.H. Tian, L. Xu. The Modern Management of Medical Imaging Records Materials, 2014, 15(9):14-15.

[5] M.Y. He. Research and Simulation on Medical Image Enhancement Algorithm. North University of China, 2015.

[6] G.H. Liu, L. Wang, W.L. Li, Y. Li. Fractal Compression Technology of Medical Imaging. China Healthcare Frontiers, 2009, 4(17):12-13. 